Editorial

\title{
Life-span plasticity of the brain and cognition: From questions to evidence and back
}

\section{A R T I C L E I N F O}

\section{Keywords:}

Life-span

Aging

Brain

Cognition

Plasticity

Longitudinal analysis

Cognitive training

\begin{abstract}
A B S T R A C T
Experience-related changes induced by modification of environment, physical exercise, or cognitive training affect brain structure and function. Research on brain plasticity and its relationship to experiential changes gathers momentum and attracts significant public interest. This collection of papers is based on presentation at the First International Conference on Life-Span Plasticity of Brain and Behavior: A Cognitive Neuroscience Perspective that took place in Detroit, MI, on October 12-14, 2011. The conference honored Margret M. and Paul B. Baltes, the pioneers of life-span developmental psychology who initiated some of the first studies on experience- and training-related changes in cognition across the life span.
\end{abstract}

(c) 2013 Elsevier Ltd. All rights reserved.
... it may be asked whether the organs increase by exercise? This may happen in the brain as well as in the muscles. ${ }^{1}$

\section{J.C. Spurzheim, 1815}

... the phenomena of habit in living beings are due to the plasticity of the organic materials of which their bodies are composed. ${ }^{2}$

W. James, 1890

When you and I have a conversation genes are being altered in our respective brains. ${ }^{3}$

\section{E. Kandel, 2010}

The quotes at the top of this page span a period of almost 200 years, a testimony to a longstanding contemplation of the brain's sensitivity to experience. The scientific roots of this fascination can be traced to the exchange between two 18th century scientists, Michele Vincenzo Giacinto Malacarne, a chief surgeon of Turin, and his friend and frequent correspondent from Geneva, Charles Bonnet. In 1779, Malacarne wrote to Bonnet about his observation on structural differences between the cerebella of individuals with severely impaired intellectual capacity and those of their normal counterparts. Malacarne believed that neuroanatomical underdevelopment could have caused poor mental abilities, but Bonnet, turning the argument on its head, replied: "La capacité intellectuelle ne dépendroit donc pas du nombre des lamelles, mais le nombre des lamelles dépendroit de l'exercice de la capacité intellectuelle." (Bonnet, 1779) Malacarne responded to the challenge by taking two dogs from the same litter, providing one with extensive training and leaving the other without any special attention. At the

\footnotetext{
1 Spurzheim (1815), p. 546.

2 James (1890), p. 105.

3 Kandel (2010), http://www.3sat.de/delta/pdf/Interview_Kandel_englisch.pdf.

${ }^{4}$ Intellectual capacity may not, therefore, depend on the number of folia, but the number of folia may depend on exercising intellectual capacity.
}

conclusion of his experiment, Malacarne observed that the cerebellum of the trained dog exhibited more numerous folia than that of its untrained litter-mate (Malacarne, 1793, cited from Rosenzweig, 1996).

For a while, the idea of the brain being altered by experience lost its appeal in spite of being endorsed by no lesser authority than William James (1890). The experimental research on experience-related plasticity remained dormant until the seminal study by University of California scientists who observed that rats exposed to a challenging environment had larger brains than their littermates raised in standard dull cages (Bennett et al., 1964). Since then, research has demonstrated that training, physical and cognitive, significantly alters the basic structure of the mature brain as it affects synaptogenesis, neurogenesis, and angiogenesis (Renner and Rosenzweig, 1987; van Praag et al., 2000). In the past decade, manipulations of physical activity regimens, cognitive training, and intense scholarly activity have been shown to correlate with structural brain alterations in humans as well (cf. Voss et al., 2013). However, the neurobiological meaning of modifications in brain structure in response to physiological, cognitive or behavioral change remains obscure.

In most general terms, plasticity is "the quality or state of ... capacity for being molded or altered, but also "the ability to retain a shape attained by ... deformation" (Merriam-Webster Dictionary; http://www.merriam-webster.com/dictionary/plasticity; accessed August 30, 2013). The Ancient Greek source or of the term, $\pi \lambda \alpha \sigma \tau o ́ \zeta$, means molded, formed, shaped, but also fake, bogus, and counterfeit, a meaning that became primary in Modern Greek (http://www.wordreference.com/gren/\%CF\%80\%CE\%BB\%CE\%B1\% CF\%83\%CF\%84\%CF\%8C\%CF\%82, accessed August 19, 2013). As the findings of significant changes in brain anatomy attributed to various experiences and regimens of training accumulate, and the discussion of plasticity is capturing public imagination (e.g., Begley, 2007), 
it becomes imperative to examine this complex phenomenon, while diligently separating the true and reliable from the spurious, transient and outright false.

This special issue on neural and cognitive plasticity from a life-span perspective is based on presentations and discussions at the First International Conference on Life-Span Plasticity of Brain and Behavior: A Cognitive Neuroscience Perspective that took place in Detroit, MI, on October 12-14, 2011. The conference, generously sponsored by the Margret $\mathrm{M}$. and Paul B. Baltes Foundation (Germany; http://www.margretbaltes-stiftung.de/Englishwebsite/Englishhome.htm) and the Institute of Gerontology at Wayne State University, commemorated the life work of two scientists whose ideas shaped modern gerontology, and whose observations on the life experiences of aging individuals has helped to spur interest in the malleability of human behavior and cognition throughout the life span.

Margret Baltes' meticulous observational studies significantly contributed to our understanding of behavioral plasticity in late adulthood. In her studies of behavioral change in nursing homes, she documented how a "dependence-support, independence-ignore" interaction script can radically change seemingly stable, life-long patterns of behavior within the course of a few weeks (Baltes, 1988, 1995; Baltes and Baltes, 1982). Margret Baltes' work shows that plasticity is a fundamental quality of the aging organism, as it enables changes in response to environmental demands, though not always for the better.

Paul Baltes contributed to the understanding of cognitive plasticity by conceptualizing the problem and conducting seminal training studies on memory and fluid intelligence. Notably, throughout his career, he emphasized the need to study individual variations in cognitive change across the entire life span and promoted a bio-cultural understanding of cognitive plasticity. Baltes most lucidly and consistently asserted that developmental trajectories as observed in the wild are not the immutable expression of human nature but malleable bio-cultural products, whose upper boundaries should be explored by systematic variation of developmental contexts (Baltes et al., 2006). Baltes viewed plasticity as a source of individual differences and an engine of developmental and societal change.

Margret and Paul's work was conducted before noninvasive neuroimaging became a routine tool of developmental research. Thus, we will never know what structural and functional changes would have been observed in the participants of their studies. The advent of noninvasive imaging techniques allowed such inquiry to be conducted routinely, and within less than a decade, studies of brain correlates of cognitive plasticity matured sufficiently to merit an interim summary. Providing such a summary was the objective of The First Baltes Conference in Detroit. In this series of articles that originated from the presentations at that conference, the authors review evidence on cognitive plasticity across the life span, with an emphasis on neuroanatomical, metabolic, and neurochemical responses of the brain to experience, in general, and to systematic cognitive and physical interventions, in particular.

In her analysis of life-span differences in cognitive plasticity, Li (2013) focuses on dopamine (DA), arguably one of the most significant neurotransmitter systems of the mammalian brain. She emphasizes the role of the DA-driven reward system in life-span development, noting the affinity between the inverted-U curve of age differences in DA and age differences in cognitive plasticity, both peaking at adolescence and declining in senium. Li emphasizes the multiple factors from which life-span trajectories of brain and cognitive development are co-constructed, including not only influence of genetic variants, but age $\times$ gene and even age $\times$ gene $\times$ gene interactions, as well as multiple environmental factors such as stress (McEwen and Morrison, 2013), which may bend and stretch developmental trajectories. It is not by chance that the DA system attracted attention in the discussion of plasticity, especially with reference to training of cognitive skills. In this context, plasticity is viewed primarily as a means of inducing beneficial change in a stable structure and maintaining the resulting gains over time. We are reward-craving creatures, so linking change to the reward system may be especially beneficial. The premier role of DA in reward and executive control processes make it a likely agent of cognitive change.

The role of DA in cognitive and functional plasticity is developed further by Bäckman and Nyberg (2013) (B\&N, 2013). B\&N focus on a specific ability (working memory) and the fronto-striatal dopaminergic network associated with this ability, and of changes in both. They emphasize a non-unitary nature of WM that calls for systematic training of its component processes, with customtailored tests for transfer of training. They stress that successful transfer should engage brain systems that are similar to those that are changed by the trained tasks. An important contribution of their review is in broadening the focus of the extant plasticity literature from the cortex, a site of structural changes revealed by several studies on experience-related plasticity, to extra-cortical structures, and especially, the striatum. B\&N use extensively what is essentially a Mendelian randomization approach, with individual variations in dopamine levels and dopamine receptor activity represented by multiple genetic variants in relevant DA genes. The review finds that WM training works well in the young, who show dose-response effects and a certain degree of transfer to similar but different cognitive tasks. However, they document very limited if any success in the old. In accord with Li, they link age-related decrements in the ability to induce change by training to age-associated decline in several aspects of the DA system. One implication of their review is that manipulating the declining DA system may hold promise for extending benefits of cognitive training into late adulthood.

If anyone inquires during what period of the life span one should start studying plasticity, the answer is clear - from the very beginning of development. Embryogenesis is arguably most plastic developmental stage, and the theoretical importance of incorporating that stage in the investigation of experience-related plasticity can be hardly overstated. Fetal development unfolds in a narrow time window with remarkable speed and precision. Thus, plasticity-related gains must be highly visible and detectable in a short period. Fetal imaging, as reviewed by Anderson and Thomason (2013) (A\&T, 2013) has generated high hopes for future research. With progress in measurement procedures that enable noninvasive monitoring of fetal brain activity and structure in utero, it is has become increasingly likely that we will be able to find out how this activity, functional connectivity, and structure are shaped by direct (mainly auditory and vibratory) stimulation or by spontaneous activity of the fetus itself. The importance of the latter should not be overlooked, for as recent studies show (Freund et al., 2013), spontaneous interactions with the environment give rise to significant differences even among genetically identical organisms. This interactive individuation process probably starts with intrauterine activity of the fetus, and it is exciting to have tools that allow capturing at least some aspects of this rapid expression of the organism's plasticity.

Rodent studies demonstrate greater structural plasticity in younger animals. Such age-related difference makes sense in the context of high demands on behavioral change in developing organisms. Lourenco and Casey (2013) (L\&C, 2013) review the recent advances in developmental neuroscience and examine plasticity as an engine of development, an idea that has been promoted by Paul Baltes. It indeed seems advantageous for a young individual to expand its behavioral repertoire, and to map sources of reward and danger in a reasonably wide swath of the environment. L\&C summarize research findings that support the notion of high tolerance 
for ambiguity among adolescents. Such built-in tolerance for multiple options may underlie the gradual emergence of individuality (Freund et al., 2013). However, therein lurks a paradox: openness to multiple options perceived as similar in reward potential may make selection more difficult, especially when the prefrontal cortices are not yet fully developed. Thus, the effects of plasticity on development are multifaceted and interactive rather than uniformly good.

An intriguing line of inquiry that developed in the past two decades suggests that cognitive improvement can be induced by non-cognitive manipulations. Physical training may hold the promise of enhancing cognitive performance by altering its neuroanatomical substrate. Hötting and Röder (2013) (H\&R, 2013) review the literature on the impact of acute and chronic physical training programs, such as aerobic exercises, resistance training, low-impact and low-effort stretching on cognition. While lauding the attained progress, H\&R identify a serious limitation of this literature: most of the extant studies test the effects of intervention on single cognitive tests, sometimes selected a priori, but not infrequently picked a posteriori based on statistical significance. Thus, little is known about the impact of various modes of physical intervention on cognitive performance assessed at the construct level, that is, at the level of cognitive abilities. If intervention effects are observed on one cognitive task but not on the other, it does not necessarily mean that the finding is specific or selective; it may point to a chance event. A relative dearth of information on the basic psychometric properties of the tasks used in the reviewed literature makes interpretation of the results even more difficult. The review also raises an important question regarding the putative mechanisms underlying cognitive change associated with physical interventions. How much of the effect is due to improved cardiovascular fitness, reduced vascular risk factors, or systemic or brain-specific effects on BDNF and other neurotrophins? The review lists very few studies in children, and almost no life-span comparisons. With a new epidemic of childhood obesity that is associated with reduced mobility and negative changes in all of the suggested mechanisms as well as threats to cognitive development, a life-span approach to physical intervention may be more important than ever.

Ofen and Shing (2013) (O\&S, 2013) provide a concise review on the life-span development of memory, which is marked by a gradual decline in perceptual-speed aspects of memory and crystallization of semantic memory as a separate system that exerts progressively increasing influence over the episodic memory. The O\&S review suggests that the developmental trajectories of memory and brain systems are less than perfectly synchronized. It is possible that not only the timing of brain maturation affects the timing of shifts from perceptual to semantic domination of mnemonic activity, but emergence of a mature modes of cognitive activity influence brain development. These differences in developmental timing may have implications for training memory at different ages. The authors stress the role of highly plastic parts of the brain (e.g. the dentate gyrus, DG, in pattern separation) and of age-sensitive regions such as the prefrontal cortex (PFC) in strategic support that differ in importance for different aspects of memory. The review raises the question whether development of strategic and associative memory systems may be driven by different types of plasticity, such as neurogenesis in the DG, synaptogenesis and angiogenesis in the PFC, and modified by stress-related remodeling in both regions.

Does every physical intervention hold an equal potential to alter brain and cognition? Voelcker-Rehage and Niemann (2013)(VR\&N, 2013) draw a distinction between "metabolic exercise (ME)" and "coordinative exercise (CE)." ME involves the use of previously acquired motor skills to boost metabolic activity of the system and to alter system energetics. Exemplary representatives of ME are resistance training, running, and cycling. $\mathrm{CE}$, on the other hand, is about teaching new skills and expanding the repertoire of action; most perceptual-motor and cognitive training programs fall under that rubric. Another important distinction that VR\&N present is fitness vs. training studies. This distinction is a variant on the core problem of developmental research: reconciling cross-sectional assessment of individuals (a snap-shot of fitness) with longitudinal evaluation of change, that is, the results or correlates of training. The VR\&N review stresses the need for further studies to examine the role of various types of tissue in gross volume change observed on MRI. One study (Thomas et al., 2012) attempts to shed light on 2013 but more are needed to establish whether angiogenesis, glial proliferation, or changes in neuron size and number account for the observed experience-related changes.

Finally, Lövdén et al. (2013) (2013) review the extant literature on anatomical plasticity in healthy adults as it is expressed in changes observable on Magnetic resonance Imaging (MRI) scans. They catalog findings from studies of experience-related change in local brain volume, cortical thickness, gray matter density, and diffusion properties, evaluate the outcomes and limitations of the studies, and propose a framework or agenda for future research. Lövdén and colleagues point out that many of the available studies of human brain plasticity lack focused regional predictions, do not show clear associations between structural change and cognitive or physiological gain, and, when they do show such association, fail to examine its dose-response characteristics. Beginning with the landmark paper on brain response to training (Draganski et al., 2004), most studies in this area use VBM for assessment or neuroanatomical change. The authors emphasize that focal findings from VBM have unclear correspondence to underlying neurobiology although, as shown by Kennedy et al. (2009), they may reflect volumetric changes on the level of gross anatomical structures.

What is the neurobiology behind MRI findings of change? Assessment of changes in cortical thickness and volume of specific, well-defined neuroanatomical regions such as the hippocampus (Erickson et al., 2011) or cerebellum (Raz et al., 2013) indicates that aerobic training and cognitive task practice can be linked to measurable change in volume. Even when observed, changes in brain structure are not easy to interpret. For example, what do the observed increases in hippocampal volume (Erickson et al., 2011) or the lack of cerebellar shrinkage (Raz et al., 2013) mean? Structural MRI morphometry is a blunt instrument and we have little notion of the degree to which changes in brain volume observed with MRI reflect neurobiological processes, and if they do, which ones. If indeed experience-related changes are mainly in the number and location of synapses as the reviewed studies indicate, then gross changes in volume and cortical thickness should represent something else. One of the candidates is angiogenesis and increase in other supporting non-neural tissue, such as astrocytes. It is possible that learning-induced proliferation of synapses and dendritic branching results in expansion of supporting tissue in response to the increased energetic demand. Measuring local change in blood volume and energy consumption during and after cognitive or physical interventions may provide clues to the nature of experience-related increase in local volume.

Lövdén and colleagues emphasize the importance of improving the methods that allow assessment of change in white matter, which may prove more responsive to cognitive interventions than cortex is. Unlike neurons, which in adult humans are produced de novo only in the dentate gyrus, glial cells are routinely generated in the adult brain. However, it is unclear if production of new oligodendrocytes or astrocytes can explain changes in MRI-derived local volumes. Myelin regeneration around an injured axon is often incomplete and does not prevent demise of the neurons that the axon connects, and astrocytes are produced only in limited amount (Bergmann and Frisén, 2013). 
This series of reviews covers a wide swath of the extant literature and contributes to setting the agenda for the near future. The readers will draw their conclusions, but here are a few thoughts inspired by the contributions to this special issue.

Development is not for children only. We need to become more serious about the life-span approach to change and acknowledge that development is not just child development but a life-long process. Early life experiences put significant constraints on later development and may result in qualitatively different responses to identical environments in adulthood (Kolb et al., 2003). This may sound obvious and even trivial, but the dearth of life-span studies on the brain, cognition, and their trajectories of change suggests otherwise. Religious, political, and ethical arguments aside, the span of time in which plasticity is to be investigated should include the in utero stage.

The challenge of change. If we are impressed by the seemingly endless variety of human anatomy, physiology, and behavior, we should continue cross-sectional investigations and describe individual differences, such as an amazing array of indicators on which physically fit people differ from their sedentary counterparts and expert musicians or taxi drivers show a stark contrast to the tonally challenged and navigationally insecure public. However, those of us who claim interest in change, as well as individual differences in change, should abandon cross-sectional paradigms as lacking any significant informative value for understanding development and devote our time and efforts to longitudinal studies. Measuring change is not a matter of comparing the states of "before" and "after." Many skillful and talented people (e.g., Rogosa and Willett, 1985; McArdle and Nesselroade, 1994) have spent some time on developing sophisticated methods of measuring change and assessing individual variability therein, and it behooves us to reap the fruits of their work. Getting the most out of latent variable analysis methods to study brain and cognitive plasticity requires reliable measures, sufficiently large samples, and multiple measurement occasions. In other words, it is going to be expensive; and worth it. It is not enough to establish the pre- to post-training change in cognitive skill and its relation to the change in observable brain parameters. Multiple measurements are needed to chart the trajectory of retention and dissipation of gains, as well as their response to suppressors and boosters. Does training repeated after a significant delay follow the same course and elicit the same brain changes as it did the first round? For example, we do not know for how long experience-induced changes remain, under what conditions are they maintained, strengthened or weakened, and whether retention of acquired skill necessitates preservation of brain changes at all, and if so, which ones.

If there is some progress in uncovering the associations between experience-related structural and cognitive change, little is known about the relationship between functional and structural changes. The vast majority of fMRI and PET studies of task-related brain activation is cross-sectional and inform only about age differences. Many report that in older adults, the tasks that engage various non-frontal circuits, elicit increased prefrontal activation (Di et al., 2013). However, when change is examined across two occasions, older adults reveal reduction of activation in the prefrontal cortex on a working memory task (Nyberg et al., 2010). This discrepancy between cross-sectional and longitudinal findings poses a challenge to the investigations of brain plasticity in middle-age and older adults. The questions of conditions under which repeated use of a specific brain circuitry leads to a structural change, modulation in functional activation, or both, and of the relationship between the two remain largely unexplored. The questions of how the magnitude of change in structure and activation is related to the behavioral change and how experience- and training-related changes fit into the general pattern of age-related decline and preservation also await thorough exploration.
Tasks and targets. In establishing experience-induced effects on brain structure, we have to navigate a narrow path between generalizability and specificity. On the one hand, as stated in a recent review, training is usually not conducted on tasks that are specific to a target brain area they are supposed to change (Thomas and Baker, 2013). Thus, we do not know whether interventions shape the brain directly or bring into action other factors that do the job. On the other hand, a specific task is not equivalent to a construct. One test of working memory, no matter how reliable and valid, is not the same as Working Memory, a construct defined by multiple measures. Therefore, for each cognitive training study, we need first to establish (or use previously established) constructs under consideration, train people on multiple indicators of these constructs, and then test learning and its effects on the brain at the level of the construct (for a recent example, see Schmiedek et al., 2010). If one memory test correlates with brain changes and the other does not, it may be evidence of superior sensitivity of that task to training, but, more likely, it may reflect capitalization on chance. Alternately, manipulation of experimental variables within a given task may allow tapping into associations between improvement in specific cognitive operations and changes in specific, predicted brain locations, as demonstrated by a recent elegant study in a rodent model of spatial navigation (Lerch et al., 2011). We need to examine training, transfer, and neuroanatomical consequence thereof on the level of constructs rather than task-specific skills. For, as Spurzheim (1815) put it: "to exercise is synonymous with putting in action: hence in exercising one faculty, we do not exercise another, and every faculty must be exercised in itself." (p. 547).

From voxels to biology. Pictorial renditions of brains are fascinating to look at. However, the goal is to understand the links between the voxels and the neurobiological "wetware" that drives behavior and changes with it. Thus, we need further studies to examine the role of various types of tissue in gross volume change observed with MRI. It is important to establish whether angiogenesis, glial proliferation, or changes in neuron size and number account for the observed experience-related change (cf. Thomas et al., 2012). We need more animal model studies in which serial MRI images are correlated with multiple-occasion assessments of learning. Some important steps have been made in this direction (Lerch et al., 2011), though neuroimaging limited to ex vivo single-occasion measurements is insufficient for establishing neural correlates of MRI-observed change. Further developments in MRI technology and sequence design are needed to match the exquisite resolution obtained in that study while allowing repeated in vivo scans.

Location, location... To date, most of the studies reviewed in this Special Issue have concentrated on the cerebral cortex (with some notable exceptions, e.g., Erickson et al., 2011; Raz et al., 2013), as the chosen methods of MRI data analysis frequently preclude examination of subcortical nuclei and the cerebellum. When the basal ganglia and the cerebellum are examined, it is usually in the context of motor skill acquisition. As it is clear from the past two decades of research, cerebellum is not a strictly motor structure and plays important role in various cognitive tasks (see Timmann and Daum, 2007, and Koziol et al., 2013, for reviews). Studies of experience- and training-related plasticity in the striatum and cerebellum structure as well $\mathrm{CBF} / \mathrm{CBV}$ changes are especially rare in children.

The "dark side of plasticity." Plasticity is just a property of the central nervous system, and it would be unwise to ascribe to it only curative or positive roles. "[Plasticity is the mechanism for development and learning, as much as a cause of pathology and the cause of clinical disorders..." (Pascual-Leone et al., 2005; p. 396). Plasticity may be a response to rewarded behavior, a change in maintenance of previously acquired behaviors, or just a by-product of activity (Wolpaw, 1997). Plenty of examples illustrate the dark side of this potentially beneficial phenomenon: focal dystonia in 
musicians (Classen, 2003); tinnitus (Pantev et al., 2012), poor recovery from spinal cord injury (Brown and Weaver, 2012) are all laid at the feet of plasticity, and judging by the distribution of agerelated differences in the brain, regions that retain greater plasticity may also be especially prone to age-related vulnerability (Raz et al., 1997; 2001). Thus, while the research efforts concentrate on finding ways to maintain and promote plasticity throughout the adult life span, more attention should be paid to unintended consequences of manipulation of plasticity in adulthood. For example, if the brakes on plasticity are lifted (Hensch, 2004; Bavelier et al., 2010), can they be re-engaged at will? Will older adults, whose capacity to enjoy experience-related plasticity is heightened through enhancing their DA reward system to adolescent levels, also re-experience the less than salubrious sides of adolescence, this time with a frailer body? Will immersion in video games restructure adult lives and alter their balance of social and physical activities? Chronic stress impairs functional and structural neuroplasticity (McEwen and Morrison, 2013), and mere progression through life increases the likelihood of encountering stressful experiences. Thus, to some extent, reduction of plasticity may protect against stress-induced changes and unsustainable metabolic cost. Hence, the direction of influence exerted by promotion of plasticity at older age needs to be examined in that context.

This is just a selection of the questions that come to mind. The inquiry into brain plasticity, its life-span trajectories and its role in shaping cognition from cradle to grave has just begun. One can only hope that it will continue gaining momentum, enriching the understanding of the intricate interactions between brain structure and mental as well as physical aspects of experience in our complex environment.

\section{Acknowledgements}

NR is supported by the National Institutes of Health grant R37AG11230. UL is supported by the Max Planck Society and the Gottfried Wilhelm Leibniz Prize 2010 of the Deutsche Forschungsgemeinschaft.

\section{References}

Anderson, A.L., Thomason, M.E., 2013. Functional plasticity before the cradle: a review of neural functional imaging in the human fetus. Neurosci. Biobehav. Rev. 37, 2220-2232.

Bäckman, L., Nyberg, L., 2013. Dopamine and training-related working-memory improvement. Neurosci. Biobehav. Rev. 37, 2209-2219.

Baltes, M.M., Baltes, P.B., 1982. Microanalytic research on environmental factors and plasticity in psychological aging. In: Field, T.M., Huston, A., Quay, H.C., Troll, C., Finney, G.E. (Eds.), Review of Human Development. Wiley, New York, pp. $524-539$

Baltes, M.M., 1988. The etiology and maintenance of dependency in the elderly: three phases of operant research. Behav. Therapy 19, 301-319.

Baltes, M.M., 1995. Dependency in old age: gains and losses. Curr. Direct. Psychol Sci. 4, 14-19.

Baltes, P.B., Lindenberger, U., Staudinger, U.M., 2006. Life span theory in developmental psychology. In: Handbook of Child Psychology. John Wiley \& Sons, Inc. New York, pp. 569-662.

Bavelier, D., Levi, D.M., Li, R.W., Dan, Y., Hensch, T.K., 2010. Removing brakes on adult brain plasticity: from molecular to behavioral interventions. J. Neurosci. 30 (45), 14964-14971, http://dx.doi.org/10.1523/JNEUROSCI.4812-10.2010.

Begley, S., 2007. The Brain: How the Brain Rewires Itself, http://www.time.com/time/magazine/article/0,9171,1580438,00.html (accessed 09.01.07).

Bennett, E.L., Diamond, M.C., Krech, D., Rosenzweig, M.R., 1964. Chemical and anatomical plasticity of the brain. Science 146 (3644), 610-619.

Bergmann, O., Frisén, J., 2013. Why adults need new brain cells. Science 340 (6133), 695-696, http://dx.doi.org/10.1126/science.1237976.

Bonnet, C., 1779. A Letter to Vincenzo Malacarne, December 24, 1779. Bibliothèque Publique et Universitaire de Genève, Fonds Bonnet, 180-181, 193-194, 217, 226, 228-229, 241.

Brown, A., Weaver, L.C., 2012. The dark side of neuroplasticity. Exp. Neurol. 235 (1), 133-141, http://dx.doi.org/10.1016/j.expneurol.2011.11.004.

Classen, J., 2003. Focal hand dystonia - a disorder of neuroplasticity? Brain 126, 2571-2572.
Di, X., Rypma, B., Biswal, B.B., 2013. Correspondence of executive function related functional and anatomical alterations in aging brain. Prog Neuropsychopharmacol Biol Psychiatry 48C, 41-50, http://dx.doi.org/10.1016/j.pnpbp.2013.09.001 (Epub ahead of print)

Draganski, B., Gaser, C., Busch, V., Schuierer, G., Bogdahn, U., May, A., 2004. Neuroplasticity: changes in grey matter induced by training. Nature 427 (6972) 311-312.

Erickson, K.I., Voss, M.W., Prakash, R.S., Basak, C., Szabo, A., Chaddock, L., Kim, J.S., Heo, S., Alves, H., White, S.M., Wojcicki, T.R., Mailey, E., Vieira, V.J., Martin, S.A., Pence, B.D., Woods, J.A., McAuley, E., Kramer, A.F., 2011. Exercise training increases size of hippocampus and improves memory. Proc. Natl. Acad. Sci. USA 108 (7), 3017-3022, http://dx.doi.org/10.1073/pnas.1015950108.

Freund, J., Brandmaier, A.M., Lewejohann, L., Kirste, I., Kritzler, M., Krüger, A., Sachser, N., Lindenberger, U., Kempermann, G., 2013. Emergence of individuality in genetically identical mice. Science 340 (6133), 756-769, http://dx.doi.org/10.1126/science.1235294.

Hensch, T.K., 2004. Critical period regulation. Annu. Rev. Neurosci. 27, 549-579.

Hötting, K., Röder, B., 2013. Beneficial effects of physical exercise on neuroplasticity and cognition. Neurosci. Biobehav. Rev. 37, 2243-2257.

James, W., 1890. The Principles of Psychology. Vol. I. of 2 vols. Henry Holt, New York.

Kandel, E., 2010. Interview on German SAT3 TV Channel ("Feuer der Erinnerung Der Medizin-Nobelpreisträger Eric Kandel im Gespräch mit Gert Scobel in Berlin"), English version: http://www.3sat.de/delta/pdf/Interview_Kandel_englisch.pdf

Kennedy, K.M., Erickson, K.I., Rodrigue, K.M., Voss, M.W., Colcombe, S.J., Kramer, A.F., Acker, J.D., Raz, N., 2009. Age-related differences in regional brain volumes: a comparison of optimized voxel-based morphometry to manual volumetry. Neurobiol. Aging 30, 1657-1676, http://dx.doi.org/10.1016/j.neurobiolaging.2007.12.020.

Kolb, B., Gibb, R., Gorny, G., 2003. Experience-dependent changes in dendritic arbor and spine density in neocortex vary qualitatively with age and sex. Neurobiol. Learn. Mem. 79 (1), 1-10.

Koziol, L.F., Budding, D., Andreasen, N., D'Arrigo, S., Bulgheroni, S., Imamizu, H., Ito M., Manto, M., Marvel, C., Parker, K., Pezzulo, G., Ramnani, N., Riva, D., Schmahmann, J., Vandervert, L., Yamazaki, T., 2013. Consensus paper: the cerebellum's role in movement and cognition. Cerebellum (Epub ahead of print).

Lerch, J.P., Yiu, A.P., Martinez-Canabal, A., Pekar, T., Bohbot, V.D., Frankland, P.W. Henkelman, R.M., Josselyn, S.A., Sled, J.G., 2011. Maze training in mice induces MRI detectable brain shape changes specific to the type of learning. NeuroImage 54, 2086-2095.

Li, S.C., 2013. Neuromodulation and developmental contextual influences on neural and cognitive plasticity across the lifespan. Neurosci. Biobehav. Rev. 37, 2201-2208.

Lourenco, F., Casey, B.J., 2013. Adjusting behavior to changing environmental demands with development. Neurosci. Biobehav. Rev. 37, 2233-2242.

Lövdén, M., Wenger, E., Mårtensson, J., Lindenberger, U., Bäckman, L., 2013. Structural brain plasticity in adult learning and development. Neurosci. Biobehav. Rev. 37, 2296-2310

Malacarne, M.V.G., 1793. Journal de Physique (Paris) 43, 73

McArdle, J.J., Nesselroade, J.R., 1994. Using multivariate data to structure developmental change. In: Cohen, S.H., Reese, H.W. (Eds.), Life Span Developmental Psychology: Methodological Contributions. Erlbaum, Hillsdale, NJ, pp. 223-267.

McEwen, B.S., Morrison, J.H., 2013. The brain on stress: vulnerability and plasticity of the prefrontal cortex over the life course. Neuron 79 (1), 16-29, http://dx.doi.org/10.1016/j.neuron.2013.06.028.

Nyberg, L., Salami, A., Andersson, M., Eriksson, J., Kalpouzos, G., Kauppi, K., Lind, J. Pudas, S., Persson, J., Nilsson, L.G., 2010. Longitudinal evidence for diminished frontal cortex function in aging. Proc. Natl. Acad. Sci. USA 107 (52), 22682-22686, http://dx.doi.org/10.1073/pnas.1012651108.

Ofen, N., Shing, Y.L., 2013. From perception to memory: changes in memory systems across the lifespan. Neurosci. Biobehav. Rev. 37, 2258-2267.

Pantev, C., Okamoto, H., Teismann, H., 2012. Tinnitus: the dark side of the auditory cortex plasticity. Ann. N. Y. Acad. Sci. 1252, 253-258, http://dx.doi.org/10.1111/j.1749-6632.2012.06452.x.

Pascual-Leone, A., Amedi, A., Fregni, F., Merabet, L.B., 2005. The plastic human brain cortex. Ann. Rev. Neurosci. 28, 377-401.

Raz, N., Gunning, F.M., Head, D., Dupuis, J.H., McQuain, J.M., Briggs, S.D., Thornton, A.E., Loken, W.J., Acker, J.D., 1997. Selective aging of human cerebral cortex observed in vivo: differential vulnerability of the prefrontal gray matter. Cereb. Cortex 7, 268-282.

Raz, N., 2001. Ageing and the brain. In: Encyclopedia of Life Sciences. Nature Publishing Group, London, http://dx.doi.org/10.1038/npg.els.0003375.

Raz, N., Schmiedek, F., Rodrigue, K.M., Kennedy, K.M., Lindenberger, U. Lövdén, M., 2013. Differential brain shrinkage over six months shows limited association with cognitive practice. Brain Cognition 82 (2), 171-180, http://dx.doi.org/10.1016/j.bandc.2013.04.002.

Renner, M.J., Rosenzweig, M.R., 1987. Enriched and Impoverished Environments: Effects on Brain and Behavior. Springer, New York

Rogosa, D.R., Willett, J.B., 1985. Understanding correlates of change by modeling individual differences in growth. Psychometrika 50, 203-228.

Rosenzweig, M.R., 1996. Aspects of the search for neural mechanisms of memory. Ann. Rev, Psychol, 47, 1-32.

Schmiedek, F., Lövdén, M., Lindenberger, U., 2010. Hundred days of cognitive training enhance broad cognitive abilities in adulthood: findings from the COGITO study. Front. Aging Neurosci., http://dx.doi.org/10.3389/fnagi.2010.00027.

Spurzheim, J.C., 1815. The Physiognomical System of Drs. Gall and Spurzheim: Founded on an Anatomical and Physiological Examination of the Nervous 
System in General, and of the Brain in Particular; and Indicating the Dispositions and Manifestations of the Mind, Being at the Same Time a Book of Reference for Dr. Spurzheim's Demonstrative Lectures. Baldwin, Cradock, and Joy, London.

Timmann, D., Daum, I., 2007. Cerebellar contributions to cognitive functions: a progress report after two decades of research. Cerebellum 6 (3), 159-162.

Thomas, A.G., Dennis, A., Bandettini, P.A., Johansen-Berg, H., 2012. The effects of aerobic activity on brain structure. Front. Psychol. 3, 86, http://dx.doi.org/10.3389/fpsyg.2012.00086.

Thomas, C., Baker, C.I., 2013. Teaching an adult brain new tricks: a critical review of evidence for training-dependent structural plasticity in humans. Neuroimage 73, 225-236, http://dx.doi.org/10.1016/j.neuroimage.2012.03.069.

van Praag, H., Kempermann, G., Gage, F.H., 2000. Neural consequences of environmental enrichment. Nat. Rev. Neurosci. 1 (3), 191-198.

Voelcker-Rehage, C., Niemann, C., 2013. Structural and functional brain changes related to different types of physical activity across the life span. Neurosci. Biobehav. Rev. 37, 2268-2295.

Voss, M.W., Vivar, C., Kramer, A.F., van Praag, H., 2013. Bridging animal and human models of exercise-induced brain plasticity. Trends Cogn. Sci. 17 (10), 525-544, http://dx.doi.org/10.1016/j.tics.2013.08.001.
Wolpaw, J.R., 1997. The complex structure of simple memory. Trends Neurosci. 20 588-594.

Naftali Raz*

Department of Psychology and Institute of Gerontology, Wayne State University, 87 East Ferry Street, Detroit, MI 48202, United States

Ulman Lindenberger

Center for Life Span Psychology, Max Planck Institute for Human Development, Lentzeallee 94, Berlin (Dahlem) 14195, Germany

* Corresponding author. Tel.: +1 3136642610.

E-mail address: nraz@wayne.edu (N. Raz) 